\title{
Too Many Blood Donors - Response Bias in the Swiss Health Survey 2012
}

\author{
Thomas Volken $^{a}$ Andreas Bänziger ${ }^{a}$ Andreas Buser ${ }^{b}$ Damiano Castellic Stefano Fontana ${ }^{d}$ \\ Beat M. Frey ${ }^{\mathrm{e}}$ Amira Sarraj $^{\mathrm{f}}$ Jörg Sigle $^{\mathrm{g}}$ Jutta Thierbach $^{\mathrm{h}}$ Tina Weingand $^{\mathrm{i}}$ \\ Behrouz Mansouri-Taleghani ${ }^{j}$
}

\author{
a School of Health Professions, Zurich University of Applied Sciences, Winterthur, Switzerland; \\ bBlood Transfusion Center Basel, Swiss Red Cross, Basel, Switzerland; \\ c Blood Transfusion Service Svizzera Italiana, Swiss Red Cross, Lugano, Switzerland; \\ dInter-Regional Blood Transfusion Service, Swiss Red Cross, Bern, Switzerland; \\ e Blood Transfusion Service Zurich, Swiss Red Cross, Schlieren, Switzerland; \\ ${ }^{f}$ Blood Transfusion Service Neuchâtelois et Jurassien, Swiss Red Cross, La Chaux-de-Fonds, Switzerland; \\ gBlood Transfusion Center Aargau-Solothurn, Swiss Red Cross, Aarau, Switzerland; \\ h Blood Transfusion Service Eastern Switzerland, Swiss Red Cross, Sankt Gallen, Switzerland; \\ 'Blood Transfusion Service Central Switzerland, Swiss Red Cross, Lucerne, Switzerland; \\ iSwiss Transfusion SRC, Swiss Red Cross, Bern, Switzerland
}

\section{Keywords}

Blood donation · Donors · Self-report · Response bias · Blood donor registry $\cdot$ Health survey

\section{Summary \\ Background: Data on blood donor status obtained from general surveys and health interview surveys have been widely used. However, the integrity of data on self-re- ported blood donor status from surveys may be threat- ened by sampling and non-sampling error. Our study aimed to compare self-reported blood donors (including one-time as well as regular donors) from the Swiss Health Survey 2012 (SHS) with register-based blood do- nors recorded by blood establishments and evaluate the direction and magnitude of bias in the SHS. Methods: We compared population-weighted SHS point estimates of the number of blood donors with their corresponding 95\% confidence intervals to the respective figures from blood donor registries (birth cohorts 1978-1993) and es- timates of donors based on period donor tables derived from blood donor registries (birth cohorts 1920-1993). Results: In the birth cohorts 1978-1993, the SHS-pre- dicted number of donors was 1.8 times higher than the respective number of donors based on registry data. Ad- justing for foreign and naturalized Swiss nationals that immigrated after their 18th birthday, the SHS overall}

predicted number of donors was 1.6 times higher. Similarly, SHS estimates for the 1920-1993 birth cohorts were 2.4 and 2.1 times higher as compared to registerbased estimates. Generally, the differences between SHS and register-based donors were more pronounced in men than in women. Conclusion: Self-reported blood donor status in the SHS is biased. Estimates of blood donors are substantially higher than respective estimates based on blood donor registries.

(c) 2016 S. Karger GmbH, Freiburg

\section{Introduction}

Data on blood donor status and blood donation history obtained from general surveys and health interview surveys have been widely used. Studies assessed characteristics, attitudes, beliefs and motives of blood donors and non-donors [1-4], institutional differences of blood donor regimes [5], and voluntary HIV counseling and testing [6-10].

However, the integrity of data on self-reported blood donor status from surveys may be threatened by sampling and non-sampling error. Important sources of non-sampling error include recall, acquiescence, and social desirability biases and have been repeatedly

\section{KARGER}

(๑) 2016 S. Karger GmbH, Freiburg 
reported in the context of surveys [11-21]. Hence, assessing the validity of data on blood donor status is imperative in order to assure that results based on surveys are suited to enhance epidemiological knowledge and guide public health actions.

Only few studies have assessed the validity of data on self-reported blood donor status. In a study that compared self-reported and record-linked blood donation history, blood donors correctly indicated whether they have ever donated blood. However, participants tended to overstate by a factor of 2 the number of donations they had made [22]. Furthermore, a study assessing the perception of blood donation in the general population of Germany concluded that giving blood is considered socially desirable and self-reported donor status may therefore be biased [23].

So far, no study has assessed the validity of data on blood donor status in the Swiss Health Survey (SHS). Moreover, we found no study that reported the direction and the magnitude of response bias related to data on blood donor status in health interview surveys.

In our study we aimed to compare the estimated number of blood donors (including one-time as well as regular donors) from the SHS 2012 (self-reported blood donors) with register-based blood donors recorded by the Regional Blood Transfusion Services of the Swiss Red Cross (SRC). Taking the register-based data (RBD) as gold standard, we assessed the bias in the survey-based estimated population prevalence of blood donors and reported its direction and magnitude.

\section{Material and Methods}

\section{Study Design}

The study is a methodologically focused secondary analysis of a populationbased, cross-sectional health interview survey carried out in 2012 in Switzerland.

\section{Study Population and Data}

The SHS carried out by the Swiss Federal Statistical Office (SFSO) is a nationwide survey on health status, health service utilization, and health-related behavior in Switzerland. The SHS was first conducted in 1992 and is repeated every 5 years. In 2012, a multistage probability sample was drawn of the entire permanent resident population of Switzerland after stratification by geographic region. The initial sample $(n=21,597)$ included subjects aged 15 years or older living in private households. Since age-related eligibility criteria of blood establishments do not allow blood donations before the 18th birthday, only subjects aged 18 years or older in 2011 were included in our study $(n=20,412)$.

Blood donor status, the outcome variable of this study, was assessed by response to the question 'Have you ever given blood since 1985?' Subjects in the SHS were categorized as blood donors or non-donors according to whether they reported to have donated blood or not. Both autologous and homologous blood donations were considered.

Data for validating the SHS point estimates of blood donors were derived from blood donor registries of the Regional Blood Transfusion Services of the Swiss Red Cross and Swiss Transfusion SRC. The RBD comprised all blood donors and more than 7,000,000 blood donations in Switzerland between January 1, 1996 and December 31, 2013.

Because the RBD did not include blood donations before 1996, we restricted the analyses to the birth cohorts 1978-1993 in a first step. Subjects included in this restricted SHS sample $(\mathrm{n}=4,425)$ were eligible for their first-time blood donation between 1996 and 2011.
Blood Donations of Foreign Nationals and Naturalized Swiss Nationals

In contrast to the RBD, subjects in the SHS who reported to have given blood did not necessarily give blood in Switzerland. In 2013, 23.8\% of the permanent resident population consisted of foreign nationals. Hence, a substantial number of foreign nationals or naturalized Swiss nationals may have given blood in their home countries, and it is therefore essential to account for blood donors who immigrated after their 18th birthday.

In order to account for blood donations outside Switzerland, we constructed weights using information from two different sources, assuming similar behavior of Swiss and foreign subjects with regard to blood donation. On the one hand, we constructed gender-specific first-time blood donor tables for the eligible population in Switzerland using the RBD from 2003 to 2013. First-time blood donation in the population was conceived as a single decrement process spawning the age interval between 18 and 65 years, and age-specific and gender-specific probabilities of first-time blood donations were derived. On the other hand, we derived the average proportion of returning blood donors from previous research [24], assuming that 70\%, 50\%, 40\%, 30\%, 20\%, 10\%, 5\%, 2.5\% and $1.25 \%$ of first-time donors will give blood $1-9$ years after their first donation and stop to donate thereafter.

Age- and gender-specific weights for foreign and naturalized Swiss nationals who immigrated after their 18th birthday were then constructed by dividing the number of first-time and returning blood donors in Switzerland by the total number of donors in home and host country. More specifically, we built the weight's nominator by adding the cumulated number of expected first-time blood donors $\mathrm{d}$ in the age interval $\mathrm{x}$ to $\mathrm{x}+\mathrm{n}$, starting at immigration age $\mathrm{k}$ and ending at age $\mathrm{K}$ in 2011 , to the cumulated proportion of donors who started their donor career outside Switzerland at age $\mathrm{k}^{*}$ and were expected to return for a further donation in the age interval $S(x, k)$ at immigration age $k$. In the denominator, all first-time donors $\mathrm{d}$ who started their donor career outside Switzerland from age 18 to the age 1 year before immigration $\mathrm{k}-1$, were added to the previous term, in order to account for the total expected ever donors in Swiss and foreign contexts.

$$
\omega_{k}=\frac{\sum_{x=k}^{K}{ }_{n} d_{x}^{G}+\sum_{x=k^{*}}^{k-1} d_{x}^{G} \cdot S(x, k)}{\sum_{x=18}^{k-1}{ }_{n} d_{x}^{G}+\sum_{x=k}^{K}{ }_{n}^{G}+\sum_{x=k^{*}}^{k-1} d_{x}^{G} \cdot S(x, k)}
$$

\section{First-Time Blood Donor Table-Based Estimates of Donors in the Birth} Cohorts 1920-1993

In a second step, we extended the analyses to the full SHS sample. Again, information from the gender-specific first-time blood donor tables and information on the proportion of returning blood donors were used to estimate the number of donors since 1985. The eligible population in the 1985-2011 period comprised all permanent residents of the birth cohorts 1920 and younger who were between 18 and 65 years old at the time of their blood donation. Consequently, members of the 1920 birth cohort were able to donate up to the end of 1985 , the year when they turned 65 , i.e., they gave blood for the first time in 1985 or they returned in 1985 for a further donation after an initial donation between 1976 and 1984 .

In order to estimate the number of subjects who gave blood at least once since 1985, we obtained data of permanent residents of Switzerland between 1976 and 2011 from the SFSO. First-time donors were estimated by multiplying the number of subjects $\mathrm{N}$ in the population age interval $\mathrm{x}$ to $\mathrm{x}+\mathrm{n}$ in year $\mathrm{t}$ by the probability of a first-time blood donation $\mathrm{q}$ in the age interval $\mathrm{x}$ to $\mathrm{x}+\mathrm{n}$. The expected number of returning first-time donors over a 10-year-period was estimated using the proportion of returning first-time donors in the interval $\mathrm{S}(\mathrm{t}, 1985)$ from the point of the first-time donation $\mathrm{t}$ up to 1985.

$$
\widehat{D}=\sum_{t=1976}^{1984} \sum_{x=18}^{X}\left[{ }_{n} N_{x}^{G}(t) \cdot{ }_{n} q_{x}^{G} \cdot S(t, 1985)\right]+\sum_{t=1985}^{2011} \sum_{x=18}^{65}{ }_{n} N_{x}^{G}(t) \cdot{ }_{n} q_{x}^{G}
$$


Table 1. Blood donors 1996-2011 in the birth cohorts 1978-1993, SHS 2012 and RBD 1996-2011

\begin{tabular}{|c|c|c|c|c|c|}
\hline \multirow[t]{2}{*}{ Nationality } & \multirow[t]{2}{*}{ RBD, N } & \multirow[t]{2}{*}{ SHS A, N (95\% CI) } & \multirow[t]{2}{*}{ SHS B, N (95\% CI) } & \multicolumn{2}{|c|}{ SHS:RBD ratio } \\
\hline & & & & A $(95 \% \mathrm{CI})$ & B (95\% CI) \\
\hline All & 121,774 & $244,830(220,543-269,117)$ & $214,873(192,862-236,884)$ & $2.0(1.8-2.2)$ & $1.8(1.6-1.9)$ \\
\hline Foreign & & $69,108(54,935-83282)$ & $39,357(29,660-49,053)$ & & \\
\hline Swiss & & $175,722(155,889-195,554)$ & $175,517(155,694-195,339)$ & & \\
\hline \multicolumn{6}{|l|}{ Women } \\
\hline All & 107,826 & $167,854(148,925-186,783)$ & $144,981(128,039-161,922)$ & $1.6(1.4-1.7)$ & $1.4(1.2-1.5)$ \\
\hline Foreign & & $47,559(36,356-58,763)$ & $26,389(18,741-34,036)$ & & \\
\hline Swiss & & $120,295(104,970-135,619)$ & $118,592(103,437-133,747)$ & & \\
\hline \multicolumn{6}{|l|}{ Total } \\
\hline All & 229,600 & $412,684(382,138-443,229)$ & $359,854(332,286-387,422)$ & $1.8(1.7-1.9)$ & $1.6(1.4-1.7)$ \\
\hline Swiss & & $296,016(271,109-320,924)$ & $294,109(269,311-318,906)$ & & \\
\hline
\end{tabular}

Confidence intervals for the estimated number of donors and confidence intervals for the probability of a first-time blood donation were derived and calculated using the methods developed by Chiang [25].

\section{Adjusting First-Time Blood Donor Table-Based Estimates of Donors for Mortality}

In a final step, the first-time blood donor table-based estimates of the number of donors since 1985 were adjusted for age- and gender-specific survival in order to achieve maximum comparability with the SHS. Obviously, subjects who participated in the 2012 SHS were alive and could report whether they donated blood or not. However, this can neither be taken for granted for subjects recorded in the RBD nor for the donor table-based estimates of donors, i.e., we do not know whether these subjects were still alive in 2012 and could report their blood donation history. Since we included birth cohorts from 1920 onwards in the analyses, a substantial proportion of donors may have deceased. Hence, we estimated the number of surviving donors by weighting the expected number of donors by the cohort age- and gender-specific probability of surviving $\pi$ from age $x$ in the year of the initial donation $t$ up to the year 2012 [26].

$\widehat{D}^{*}=\sum_{t=1976}^{1984} \sum_{x=18}^{X}\left[{ }_{n} N_{x}^{G}(t) \cdot{ }_{n} q_{x}^{G} \cdot S(t, 1985) \cdot{ }_{T} \pi_{t, x}^{G}\right]+\sum_{t=1985}^{2011} \sum_{x=18}^{65}{ }_{n} N_{x}^{G}(t) \cdot{ }_{n} q_{x}^{G} \cdot{ }_{T} \pi_{t, x}^{G}$

\section{Statistical Analyse}

We compared population-weighted SHS point estimates of the number of blood donors with their corresponding 95\% confidence intervals to the respective figures from the blood donor registries of the Regional Blood Transfusion Services of the SRC and Swiss Transfusion SRC and the first-time blood donor table-based estimates of donors. We used Stata 12.1 (StataCorp, College Station, TX, USA) for all statistical analyses. Statistical significance was established at $\mathrm{p} \leq 0.05$.

\section{Results}

In a first step, we compared RBD- and SHS-derived blood donors - unadjusted (SHS A) and adjusted (SHS B) for blood donations outside Switzerland - in the youngest birth cohorts 19781993 , i.e., in the birth cohorts for which the RBD recorded all do- nations since 1996 (table 1). In the birth cohorts 1978-1993, the unadjusted SHS A overall predicted number of subjects who gave blood at least once since 1996 was 412,684, whereas the respective number of donors based on the RBD was 229,600. Adjusting for foreign and naturalized Swiss nationals who immigrated after their 18 th birthday, the SHS B overall predicted number of donors was 359,854 . The number of RBD donors was substantially lower than the lower boundary of the $95 \%$ confidence interval of unadjusted and adjusted SHS donors (SHS A 382,138; SHS B 332,286), and the adjusted predicted overall number of SHS B donors was 1.6 times higher than the respective number of RBD donors. Similarly, the number of RBD female $(107,826)$ and male $(121,774)$ donors was substantially lower than the lower boundary of the $95 \%$ confidence interval of female and male SHS donors (SHS A/B female $148,925 / 128,039$; SHS A/B male 220,543/192,862). However, the adjusted predicted number of female SHS donors was 1.4 times higher than the number of female RBD donors, whereas the number of male SHS donors was 1.8 times higher (SHS B).

We obtained similar results when comparing SHS donors since 1985, which comprised all birth cohorts from 1920 to 1993, with estimated donors based on the RBD age- and gender-specific firsttime blood donor tables (table 2). The SHS B adjusted overall predicted number of ever donors was 1,764,258 whereas the estimated number of RBD donors was 857,202 . Again, the overall female $(398,425)$ and male $(458,777)$ number of estimated RBD donors was substantially lower than the lower boundary of the $95 \%$ confidence interval of the respective SHS donors (SHS A/B female $675,800 / 584,192$; SHS A/B male 1,254,433/1,105,064). The predicted number of SHS B donors was 2.1 (total), 1.5 (women) and 2.5 (men) times higher than the respective number of estimated RBD donors.

Adjusting RBD estimates for the expected number of deaths that occurred before the end of 2012 (table 3), the year of the SHS survey, yielded slightly higher proportions of female and male SHS 

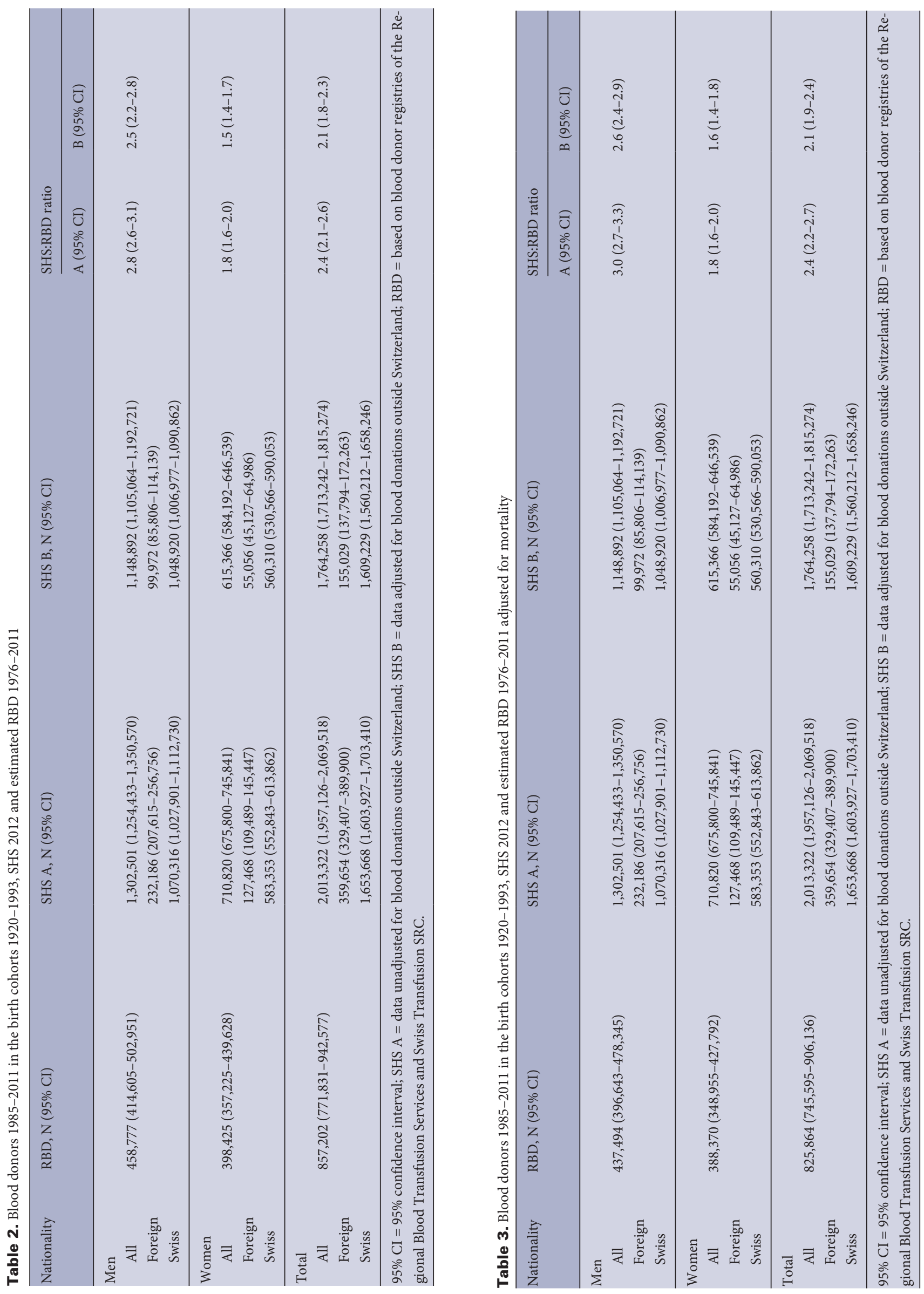
B donors (1.6 and 2.6). Again, the number of estimated RBD donors (female 388,370; male 437,494) was well below the lower boundary of the $95 \%$ confidence interval of SHS donors (SHS A/B female: 675,800/584,192; SHS A/B male: 1,254,433/1,105,064).

\section{Discussion}

All comparisons of the number of donors based on the SHS both adjusted and unadjusted for blood donations outside Switzerland - and the RBD yielded substantial differences. SHS point estimates of donors are substantially biased. The SHS B overestimates the number of donors by a factor of 1.6 in the youngest birth cohorts 1978-1993, for which the RBD recorded all blood donations. Even if we suppose that all foreign nationals and naturalized Swiss nationals exclusively gave blood in their home countries, the SHS estimates are still substantially biased. Without foreign nationals and naturalized Swiss nationals, the adjusted SHS B predicted number of Swiss donors is 294,109 as compared to $229,600 \mathrm{RBD}$ donors (table 1). Hence, the SHS overall estimates are still 1.3 times higher than the number of RBD donors. However, the RBD donors still include donors with foreign and Swiss nationality.

\section{Potential Sources of Bias}

A first source of bias which may have led to the marked differences in the number of donors between the SHS and the RBD may be related to social desirability and social approval. Studies using semantic differentials show that blood donation is very positively connoted [27-29] and is considered a socially desirable behavior [23]. Hence, respondents in the SHS may have answered the question regarding their own blood donor behavior in a more socially desirable direction and therefore induced response bias.

For both men and women, the SHS overestimated the number of donors. However, the bias for men was more pronounced than for women, and the results indicate that biases appear to vary by gender. These findings are in line with previous studies that found gender differences in social desirability and social approval bias [18, 30-32].

A second source of bias may be self-selection. Studies found that, compared to the general population, blood donors had a better self-rated health, exhibited a healthier lifestyle, and visited general practitioners and specialists less often [33]. Similarly, respondents to the second stage of the SHS were found to have a better self-rated health as compared to non-respondents [34]. Furthermore, SHS participants reported fewer inpatient hospital stays and fewer general practitioner visits than were expected on the basis of register-based health insurance data [35]. Hence, selection bias in the SHS may be induced by the over-representation of healthier subjects, which at the same time were potentially more likely to be blood donors.

The self-selection of heathier subjects in the SHS and the fact that the SHS does not include subjects living in institutions (e.g., nursing homes, hospitals) may also explain the increasing gap between SHS and RBD donors when the older birth cohorts 1920
1977 were considered in the analyses. Since older individuals in the SHS sample are presumably even healthier than younger individuals in comparison to their peers in the general population, the over-reporting of blood donors in older age groups may be even more pronounced.

Thirdly, recall bias may have led to over-reporting in the SHS, especially in older age groups. Participants were asked whether they ever donated blood since 1985. Consequently, older participants had to recall events that dated back up to 27 years. Our results suggest that the accuracy of recall may indeed be biased since the amount of bias increased when older birth cohorts were considered in the analyses. More specifically, the adjusted number of SHS donors was 1.6 times higher than the number of RBD donors in the 1978-1993 and 2.1 times higher in the 1920-1993 birth cohorts.

Finally, selection bias may have further inflated the estimated number of donors in the SHS. Several studies show that higher socio-economic status and higher education are associated with a greater likelihood of ever having given blood $[1,36,37]$. Results for the SHS 2007 indicated that subjects with lower education as well as foreign nationals were less likely to return the additional mail survey after they had completed the telephone interview [34]. It is therefore very likely that especially foreign nationals with lower education had an overall lower response rate since the interviews were conducted in German, French or Italian, which potentially favored well-integrated and well-educated subjects. Consequently, blood donors among foreign nationals may be over-represented.

\section{Implications}

The SHS is an important instrument for assessing health status, health service utilization, and health-related behavior in Switzerland. As such, its data has been used in numerous studies, which in turn help to inform and guide public health policies, public health programs, and public health initiatives. With regard to blood donation, our findings suggest that results based on self-reported blood donor status may be substantially flawed, and interpretations should therefore be approached cautiously. Potentially, our findings may be relevant for studies relying on self-reported blood donor status and assessing blood donor and non-donor characteristics and behavior [1-4], or voluntary HIV counseling and testing [6-10].

Without this list being exhaustive, several strategies are possible in order to mitigate bias in the SHS. Recall bias may be reduced by rephrasing the survey question regarding blood donation, i.e., the questionnaire should first attempt to assess whether the participant has ever donated blood and then assess the year of the last donation. The rationale behind splitting up the original question is that it is easier to recall whether one has donated blood at all than to recall whether one has donated blood since 1985 . Furthermore, to survey the year of the last donation yields more information than just assessing any donation since 1985. Self-selection bias may be mitigated by either making participation in the SHS mandatory [38] or by making data on non-respondents available in the SHS dataset. While the former strategy may not be feasible or advisable 
because the forced completion of the survey by non-compliant and possibly disgruntled participants could potentially result in even more biased data, the latter strategy would allow data analysts to specify sample selection models that aim at detecting and correcting selection bias $[39,40]$. Furthermore, several methods have been proposed to cope with social desirability bias, including the use of forced-choice items, randomized response techniques, the bogus pipeline, self-administration of the questionnaire, the use of proxy subjects, and the selection of interviewers [41].

While the above strategies may mitigate bias, it is important to keep in mind that we could only speculate on its sources. Future studies could lead to strong inferences about the nature of the bias once record-linkage between the SHS and RBD is employed. This in turn could inform effective strategy selection in order to correct for bias.

\section{Strengths and Limitations}

The main strength of our study is that it verifies the surveybased SHS estimates of blood donors in Switzerland with the number of blood donors derived from high-quality blood donor registries of the Regional Blood Transfusion Services of the SRC and Swiss Transfusion SRC.

However, several limitations should be kept in mind: Firstly, the nature of the reported bias remains unclear because record linkage between the SHS and RBD was not feasible.

Secondly, complete RBD was available for the birth cohorts 1978-1993 only. Hence RBD estimates for all eligible birth cohorts (1920-1993) had to be derived from first-time blood donor tables. However, this approach assumes that current and past birth cohorts experienced similar first-time blood donation patterns which may not necessarily be the case. Therefore, RBD estimates may be biased to some degree.
Thirdly, the blood donor history of foreign nationals and naturalized Swiss nationals remains unclear because the SHS provides no data on whether blood was donated in the country of origin or in Switzerland.

Fourthly, the SHS does not consider blood donors who emigrated. However, these donors remain in the blood donor registries. Consequently, RBD is upward biased to some degree and the difference between the SHS data and RBD could be larger than reported.

Finally, we did not consider Swiss nationals who gave blood in a foreign country but not in Switzerland since 1985. However, their number is presumably low and their potential impact on the results minimal.

\section{Conclusion}

Self-reported blood donor status in the SHS is biased. Point estimates of blood donors are substantially higher than respective estimates based on blood donor registries, and the bias is more pronounced for men than for women. While health surveys have their merits, our findings highlight the need for high-quality observational data.

\section{Acknowledgments}

This study was supported by Stiftung Lindenhof, grant number 13-04-F.

\section{Disclosure Statement}

The authors declare no conflict of interests.

\section{References}

1 Volken T, Weidmann C, Bart T, Fischer Y, Kluter H, Ruesch P: Individual characteristics associated with blood donation: A cross-national comparison of the German and Swiss population between 1994 and 2010. Transfus Med Hemother 2013;40:133-138.

$\checkmark 2$ Lemmens KP, Abraham C, Hoekstra T, Ruiter RA, De Kort WL, Brug J, Schaalma HP: Why don't young people volunteer to give blood? An investigation of the correlates of donation intentions among young nondonors. Transfusion 2005;45:945-955.

3 Robinson NG, Masser BM, White KM, Hyde MK, Terry DJ: Predicting intentions to donate blood among nondonors in Australia: an extended theory of planned behavior. Transfusion 2008;48:2559-2567.

4 Lee LC, Piliavin JA, Call VRA: Giving time, money, and blood: Similarities and differences. Soc Psychol Q 1999;62:276-290.

5 Healy K: Embedded altruism: blood collection regimes and the European Union's donor population. Am J Sociol 2000;105:1633-1657.
Houston S, Archibald CP, Strike C, Sutherland D: Factors associated with HIV testing among Canadians: results of a population-based survey. Int J STD AIDS 1998;9:341-346.

7 Hubert M, Bajos N, Sandfort T (eds): Sexual Behaviour and HIV/AIDS in Europe. Comparison of National Surveys, London, UCL Press, 1998.

8 Jeannin A, Meystre-Agustioni G, Lociciro S, DuboisArber F: Monitoring der Schweizer Präventionsstrategie gegen HIV/AIDS. Synthesebericht 2004-2008. Lausanne, Institut universitaire de médecine sociale et préventive, 2010.

9 Lemcke A, Kjoller M, Ekholm O, Smith E: HIV testing in the Danish population: a national representative survey, 2000. Scand J Public Health 2007;35:631-639.

10 Jeannin A, Dubois-Arber F, Paccaud F: HIV testing in Switzerland. AIDS 1994;8:1599-1603.

11 Lofters A, Vahabi M, Glazier R: The validity of self-reported cancer screening history and the role of social disadvantage in Ontario, Canada. BMC Public Health 2015;15:28.
12 Makimoto K, Iida Y, Hayashi M, Takasaki F: Response bias by neuroblastoma screening participation status and social desirability bias in an anonymous postal survey, Ishikawa, Japan. J Epidemiol 2001;11:70-73.

13 Moshagen M, Musch J, Ostapczuk M, Zhao Z: Reducing socially desirable responses in epidemiologic surveys: an extension of the randomized-response technique. Epidemiology 2010;21:379-382.

14 Sjostrom O, Holst D: Validity of a questionnaire survey: response patterns in different subgroups and the effect of social desirability. Acta Odontol Scand 2002; 60:136-140.

15 Sjostrom O, Holst D, Lind SO: Validity of a questionnaire survey: the role of non-response and incorrect answers. Acta Odontol Scand 1999;57:242-246.

16 Abdalla S, Abdelgadir N, Shahraz S, Bhalla K: Respondents' recall of injury events: An investigation of recall bias in cross-sectional injury data from the Sudan Household Health Survey 2010. Int J Inj Contr Saf Promot 2015;22:215-223. 
17 Hunger M, Schwarzkopf L, Heier M, Peters A, Holle R, Group KS: Official statistics and claims data records indicate non-response and recall bias within surveybased estimates of health care utilization in the older population. BMC Health Serv Res 2013;13:1.

18 Miller TM, Abdel-Maksoud MF, Crane LA, Marcus AC, Byers TE: Effects of social approval bias on selfreported fruit and vegetable consumption: a randomized controlled trial. Nutr J 2008;7:18.

19 Jordan K, Jinks C, Croft P: Health care utilization: Measurement using primary care records and patient recall both showed bias. J Clin Epidemiol 2006;59: 791-797.

20 Kruijshaar ME, Barendregt J, Vos T, de Graaf R, Spijker J, Andrews G: Lifetime prevalence estimates of major depression: an indirect estimation method and a quantification of recall bias. Eur J Epidemiol 2005;20: 103-111.

21 Lee Z, Woodliffe L: Donor misreporting: conceptualizing social desirability bias in giving surveys. Voluntas 2010;21:569-587.

22 Bertalli NA, Allen KJ, McLaren CE, Turkovic L, Osborne NJ, Constantine CC, Delatycki MB, English DR, Giles GG, Hopper JL, Anderson GJ, Olynyk JK, Powell LW, Gurrin LC, HealthIron Study I: A comparison of self-reported and record-linked blood donation history in an Australian cohort. Transfusion 2011;51:21892198.

23 Stocké V, Hukler C: Die angemessene Erfassung der Stärke und Richtung von Anreizen durch soziale Erwünschtheit. ZA Information 2004;54:53-88.

24 Volken T, Buser A, Holbro A, Bart T, Infanti L: Blood donor to inactive donor transition in the Basel region between 1996 and 2011: A retrospective cohort study. Vox Sang 2015;109:155-162.
25 Chiang CL: Statistical inference regarding life table functions; in Chiang CL (ed): The Life Table and Its Application. Malabar, Robert E. Krieger Publishers, 1984, pp 153-167.

26 Menthonnex J: La mortalité par génération en suisse. Evolution 1900-2150 et tables par génération 19002030. Rapport technique. Neuchâtel, Swiss Federal Statistical Office 2009.

27 Godin G, Sheeran P, Conner M, Germain M, Blondeau D, Gagne C, Beaulieu D, Naccache H: Factors explaining the intention to give blood among the general population. Vox Sang 2005;89:140-149.

28 Armitage CJ, Conner M: Social cognitive determinants of blood donation. J Appl Soc Psychol 2001;31:14311457.

29 Volken T, Bänziger A, Rüesch P, Maeder Net: Blutspende und Blutstammzellspende. Motive und Barrieren. Zürich, Editions à la Carte/Blutspende SRK Schweiz AG, 2013.

30 Hebert JR, Ma Y, Clemow L, Ockene IS, Saperia G, Stanek EJ, 3rd, Merriam PA, Ockene JK: Gender differences in social desirability and social approval bias in dietary self-report. Am J Epidemiol 1997;146:10461055.

31 Hebert JR, Ebbeling CB, Matthews CE, Hurley TG, Ma Y, Druker S, Clemow L: Systematic errors in middleaged women's estimates of energy intake: Comparing three self-report measures to total energy expenditure from doubly labeled water. Ann Epidemiol 2002;12: $577-586$.

32 Klein M, Kühhirt M: Sozial erwünschtes Antwortverhalten bezüglich Teilung häuslicher Arbeit. Methoden - Daten - Analysen 2010;4:79-104.

33 Atsma F, de Vegt F: The healthy donor effect: a matter of selection bias and confounding. Transfusion 2011; 51:1883-1885.
34 Volken T: Second-stage non-response in the Swiss health survey: determinants and bias in outcomes. BMC Public Health 2013;13:167.

35 Volken T, Bopp M, Rüesch P: Intensität der Inanspruchnahme von Gesundheitsleistungen in der Schweizer Bevölkerung. Auswertungen der Daten aus der schweizerischen Gesundheitsbefragung. Report commissioned by the Swiss Federal Office for Public Health. Winterthur, Zürcher Hochschule für Angewandte Wissenschaften, 2014. www.bag.admin.ch/gesundheit2020/14232/15168/index.html?lang=deðdown load $=$ NHzLpZeg7t, lnp6I0NTU042l2Z6ln1acy4Zn4Z2q ZpnO2Yuq2Z6gpJCMdnt,e2ym162epYbg2c_JjKbNoKSn6A- (last accessed September 21, 2016).

36 Veldhuizen IJ, Doggen CJ, Atsma F, De Kort WL: Donor profiles: Demographic factors and their influence on the donor career. Vox Sang 2009;97:129-138.

37 Golding J, Northstone K, Miller LL, Davey Smith G, Pembrey M: Differences between blood donors and a population sample: implications for case-control studies. Int J Epidemiol 2013;42:1145-1156.

38 Kho ME, Duffett M, Willison DJ, Cook DJ, Brouwers MC: Written informed consent and selection bias in observational studies using medical records: systematic review. BMJ 2009;338.

39 Greene WH: Sample selection bias as a specification error - comment. Econometrica 1981;49:795-798.

40 Lee BJ, Marsh LC: Sample selection bias correction for missing response observations. Oxf Bull Econ Stat 2000;62:305-322.

41 Nederhof AJ: Methods of coping with social desirability bias - a review. Eur J Soc Psychol 1985;15:263-280. 